\title{
The Impact of Food Intakes on Skin Health
}

\author{
Nasim Habibzadeh* \\ School of Health and Life Since, Teesside University, United Kingdom \\ *Corresponding Author: Nasim Habibzadeh, School of Health and Life Since, Teesside University, United Kingdom.
}

Received: October 14, 2019; Published: November 04, 2019

DOI: $10.31080 /$ ASNH.2019.03.0525

\begin{abstract}
Good nutrition is an essential part of a healthy lifestyle. Healthy food intakes will lead to an optimal health condition that initially influence the nature of skin. Foods such as wholegrains, fibre, fruit and vegetables, with limited amounts of fat, sugar and salt promote skin health. Certain foods however have bad impacts on skin perspectives. Foods with saturated fat, sugar can cause to unhealthy skin tone. Alcohol and fizzy drinks also lesions skin features. Insufficient water intakes and consuming refined products such as skimmed milk impair the skin layer. Nonetheless, using natural foods and drinks in daily consumptions and avoiding unhealthy types of diet can boost skin health and skin quality in long terms.
\end{abstract}

Keywords: Skin; Foods; Drinks; Health

\section{Introduction}

Nutrition refers the necessary foods for health and the states of being free from illnesses [1]. A healthy diet contains natural foods , plant-based nutrients, wholegrains to meet human daily requirements. A mix of proper proteins, carbohydrates, and fat and enough vitamins are the keys for optimal diet. Poor nutrition primary can result in undesired appearances in human [2].

Research shows low quality food components at the first stage exacerbate the skin integrity. Among different foods and beverages frequents drinks intakes such as alcohol dramatically affect the appearances and skin features. Per each time of drinking alcohol body gets dehydrated which initially can deplete skin blood's capillaries flow (fluids). Consequently, chronic drinking of alcohol can have detrimental impacts on skin health in long-terms [3].

Some evidence - based studies outline consuming dairy products which combined with high levels of refined foods negatively impact skin texture. It is believed that, eliminating dairy intakes products like skimmed milk, yogurt, and cheese can protect skin features from conditions such as acne and rosacea [4].
Drinking inadequate water also influence on skin hydration and its appearance. Lack of water can cause dry skin. A balanced -water consumption can help to remove toxins and any other by-products from body which improves skin tone [5]. Sufficient water intake increases fluids and liquid transitions through body.

Indeed, fizzy drinks owing to containing high levels of surge can lead to certain skin conditions such as inflamed skin or acne and avoiding of this type of drinks can preserve the skin configuration.

Saturated fats, sugars and salt foods such as pizza, chips and hamburgers notably alter skin surface as individual who often consume these type of foods look older than those individual which consume natural or conventional foods [6]. More consumptions of fish instead of meat for example significantly boost skin heath. Using natural sweets like dried fruits and dates are good substitutes for junk foods like chips for continuing skin health tone [7].

More importantly, consuming not enough fruits, vegetables are associated with ill skin health. Fruits and vegetables are foods for healthy skin and because they contain great amount of antioxi- 
dants can help to protect skin from early skin - ageing appearance [8]. The fruits and vegetables juices improve blood vessels flows which enhance skin aesthetic [9].

Nuts also include amazing health benefits on skin health. Nuts contents essential fats or fatty acids, vitamin E and B vitamins which retain skin elasticity and quality toward glowing skin. Sunflower seeds for example are a great source of vitamin $\mathrm{E}$ which can promote skin health $[10,11]$. Therefore, nuts are healthy snakes to maintain skin heath if one's can get used to it.

\section{Conclusion}

Generally, the type of diets affect skin health in different individual. Evidence proves that some foods or drinks ensure the skin health and its appearance and some other cause skin lesions. Unhealthy diets for skin are refined foods such as skimmed milk, yogurt, and cheese and foods such as pizza, chips and hamburgers. Lake of water and insufficient fruits or vegetables intakes in daily consumptions are associated with ill skin health. Heavily drinking alcohol or some other fizzy drinks dramatically impact the skin texture. Nevertheless, a proper diet that could contain a balanced amount of natural foods, fluids and fibers can significantly boost skin health and its features in all individual over time.

\section{Bibliography}

1. Danby FW. "Nutrition and acne". Clinical Dermatology 28.6 (2010): 598-604.

2. Smith RN., et al. "The effect of a high-protein, low glycemicload diet versus a conventional, high glycemic-load diet on biochemical parameters associated with acne vulgaris: a randomized, investigator-masked, controlled trial". Journal of the American Academy of Dermatology 57.2 (2007): 247-256.

3. Kostović K and Lipozencić J. "Skin diseases in alcoholics". Acta Dermatovenerologica Croatica 2 (2004): 181-190.

4. Juhl CR., et al. "Dairy Intake and Acne Vulgaris: A Systematic Review and Meta-Analysis of 78,529 Children, Adolescents, and Young Adults". Nutrients 10 (2018): pii: E1049.

5. Palma ML., et al. "Relationship between the dietary intake of water and skin hydration". Journal Biomedical and Biopharmaceutical Research 9 (2012): 173-181.

6. Habibzadeh N. "Conventional habitual nutritional changes can lead to the several health". Journal of Nutritional Biochemistry 2 (2018): 147-150.
7. Fulton J., et al. "Effect of chocolate on acne vulgaris". JAMA 210 (1969): 2071-2074.

8. Katta R and Desai SP. "Diet and Dermatology". The Journal of Clinical and Aesthetic Dermatology 7.4 (2014): 46-51.

9. Whitehead RD., et al. "Cross-cultural effects of fruit and vegetable consumption on skin color". American Journal of Public Health 102 (2012): 212-213.

10. Habibzadeh N. "Nuts, Optimum Substitutes for any Meal". Acta Scientific Nutritional Health 3 (2019): 96-97.

11. Habibzadeh N. "The Ease of Sunflower Seeds". Acta Scientific Nutritional Health 3.10 (2019).

Volume 3 Issue 12 December 2019 (C) All rights are reserved by Nasim Habibzadeh. 